\title{
A NEW METHOD OF ESTIMATION OF THE OPTIMAL AV DELAY BY USING PULSE OXIMETRY IN DDD PACED PATIENTS
}

\author{
Miroslav Měštan, Jiří Kvasnička, Pavel Rejchrt \\ $1^{\text {st }}$ Department of Internal Medicine, Charles University, Faculty of Medicine and Teaching Hospital, Hradec Králové; \\ (Head: prof. MUDr. J. Kvasnička, CSc.)
}

\begin{abstract}
Summary: The paper gives a detailed description of a new method for estimating the optimal AV delay in dual chamber paced patients, which is non-invasive, not dependent on the examinator, not time consuming and inexpensive. In principle, the pulse oximetry signal obtained by common finger probe was used to measure the change in its time course after changes in pacemaker stimulation. The Eagle 4000 monitor, manufactured by Marquette USA, was used for measurements and digitizing the data and then this data was analyzed using a portable personal computer with original programs developed in Famulus v. 3.5. Our results were compared to the standard method for optimizing AV delay which uses the direct measurement of blood pressure in the ascending aorta. Twenty-four patients with Physios pacemakers were enrolled in the pilot study. Measurements showed a positive correlation $(\mathrm{r}=0.982, \mathrm{~N}=432)$ between the changes in the pulse oximetry amplitude and changes in the pressure pulse amplitude measured in the ascending aorta by a catheter-tip manometer. These results indicated that this new method could be a possible alternative to the currently used techniques for AV interval optimization.
\end{abstract}

Key words: DDD pacing; Optimal AV interval; Pulse oximetry; Atrial contribution

\section{Introduction}

About $50 \%$ of patients indicated for pacemaker treatment have dual chamber pacemaker implanted. In this text the term DDD mode (DDDM) pacing is used only when the right atrium and the right ventricle are both sensed and stimulated. It is generally accepted that optimizing the AV interval (AVI) may be beneficial in all patients (1). Of particular importance is the optimizing of the AVI in patients with hypertrophic obstructive cardiomyopathy, dilated cardiomyopathy with severe prolongation of the AVI and in patients with left ventricular dysfunction associated with a presystolic mitral regurgitation. There is still the problem how to estimate this optimal AVI (11). The optimal AVI for the atrial synchronous pacing (DDDM) may be defined as the time interval between the atrial and the ventricular stimulus, at which the stroke volume is highest. Most currently used techniques for determining this optimal setting are either expensive or time-consuming $(2,5,4)$, and therefore are not routinely used. The aim of our study was to investigate a new, alternative method, which is easily reproducible and does not have these known disadvantages.

\section{Theoretical basis of the research}

The basis for our idea came from the experiments of Mitchell et all. in 1965 (3). Their experiments were performed in sequentially paced dogs with surgically induced AV block. They demonstrated a decrement in stroke volume in ascending aorta by a Doppler flow probe mounted on the aorta when they changed stimulation by switching off the atrial stimulus. In this way they were able to quantify the atrial contribution. In our study, we replaced the complicated direct measurement of aortic flow by an invasive direct measurement of aortic blood pressure (aBP). Keeping in mind that under these conditions, the heart rate and the mean pressure do not change and therefore there is tight correlation between the pressure pulse and the stroke volume $(8,9)$. The main idea of this paper has been to replace an invasively measured parameter by the photoplethysmographic technique, which was already tested $(4,10)$. Other studies showed that every person has only one AV delay, when the atrial contribution $(\mathrm{AC})$ is maximal.

\section{Technical equipment}

The equipment used for the chosen technique had to be common in cardiac pacing laboratories and it could not contain non standard parts, i.e. special finger oximetric probe or special software in the monitor of vital signs.

\section{The way of the pacemaker programming}

We enrolled patients with Physios dual chamber pacemakers produced by Biotronik, Germany. The programmer EPR 1000 Color (Biotronik) was used. 


\section{Technique for the monitoring of vital signs}

The monitor of vital signs Eagle 4000, Marquette USA, was used to record and digitize the ECG, aBP and oximetric signal (OS). The sampling frequency for EKG, aBP and OS was 240,120 and $60 \mathrm{~Hz}$ respectively. The monitor has three regimes for filtering and displaying curves of vital sings. The Operating room regime was chosen for our research.

\section{ECG acquisition}

The ECG curve was recorded from the II limb lead in patients who had pacemakers in right subclavian area. For patients with the pacemaker in the left subclavian area, limb lead III was used. The aim was to obtain a minimum amplitude of $1 \mathrm{mV}$ for the magnitude of the pacemaker impulse. The voltage of pacemaker impulses could be increased for the quality ECG record within the time of measurement.

\section{Blood pressure measurement}

The Millar manometer-tip catheter was used for the direct, invasive aBP recording. The monitor automatically calibrates the blood pressure curve on the screen.

Pulse oximetry measurement

Recording the pulse oximetry signal was the feature of our method. We used a common finger probe manufactured by Marquette. The monitor displayed two times the original magnitude of pulse oximetric curve.

\section{Personal computer and way of connection}

A portable personal computer with RAM of $8 \mathrm{MB}$, the Pentium processor, and hard disc capacity of $890 \mathrm{MB}$ was used for data acquisition and analysis. The computer and the monitor communicated via an ethernet connection, with the use of a PCMCIA card model Novell/Anthem NE 2000. Windows 95 was installed on the PC and programs for data analysis which were developed in MS-DOS 7.0 were initiated from the desk-top by custom icons.

\section{Patients preparation}

Patients were examined in the supine position with an elevated left upper extremity to get the finger probe higher than the chest wall thus eliminating any impact of upper extremity venous wall pressure on the OS. The Millar catheter-tip manometer was inserted into the femoral artery and advanced into the ascendent aorta under fluoroscopic guidance. The OS was recorded by a finger probe placed on a finger on the left hand.

All measurements were performed during a short period of apnea following a quiet expiration to exclude changes in the pulse amplitude of the OS caused by respiration. In this way, we maintained a constant venous return. The patients practiced this type of breathing before the measurements were actually acquired.

\section{Procedure of measurement}

During the procedure, a constant ventricular rate was maintained to exclude the impact of the „force-frequency phenomena" on the force of ventricular contractions. All patients were paced at the ventricular rate of $70 / \mathrm{min}$ or at a rate 10 beats above the resting sinus rate.

In the first part of the examination, the AVI was increased and then decreased in steps of $50 \mathrm{~ms}$. During each step the data was recorded on the hard disc of the PC.

In the second part of the examination, the pacemaker was changed from dual chamber stimulation (DDIM) to single chamber ventricular stimulation (VVIM) for 2-3 beats and then back to the DDIM stimulation. In this way, we were able to switch off the atrial stimulus.

We were interested only in the first beat after the stimulation change because of the fact that the OS amplitude quickly reverted back to its original magnitude. This quick change back can be explained in two ways. First, there can be prompt changes in a finger's microcirculation and secondly the oximetric curve is expression of the time course of relative numerical values. The oximetric devices constantly change the calibration of oximetric graph on the screen to maximize the graph on the screen. This maximization occurs within 5-10 beats.

\section{Computer analysis}

Before starting the measurement, a patient's name and heart rate was saved into a text file.

\section{Data acquisition}

Data were transferred from the monitor to the PC by the ethernet connection on-line. A special program in MSDOS using the Marquette format was developed. We did not use absolute values of ECG, aBP and OS, but rather synchronously arranged relative numerical values which exactly copied the time course of variables. The recording was started and stopped manually using a visual control on the screen of PC. The program saved data into text files after finishing each measurement. A batch program started another program for data analysis.

The main part of data analysis was performed by the Famulus 3.5 program (7). This is program for simulation and graphic presentation of mathematical and physical models. It can read and write data in text format to the hard disc and start or finish programs written in the Famulus through he use of the user's menu. A set of original programs was programmed for this measurement analysis. The programs were started by icons from the desktop section of Windows. Programs finished automatically and return to Windows.

\section{Assessment of the measurement's quality}

The main program was started after saving the data on a hard disc. Impulses of pacemaker were detected on the 
ECG record. The second derivation of ECG time course was counted and the values were corrected. The values higher or equal to 300 were corrected to 1000 value, values lower than 300 were replaced by 0 . Values where we were able to find time coordinates of impulses of pacemaker were obtained. The first stimulus and the first complete beat were located, in which the coordinates of atrial and ventricular stimulus were known, with programmed cycles with constants. The constants' values were counted from known sampling frequency of ECG, known heart rate and from five possible values of the tested AV intervals. All ECG records were tested until the moment when the time between atrial and ventricular impulses was different from that time in the first complete beat. We had to find two „pattern" beatswith the same AVI as in the first complete beat before the „tested“ beat. When we could not find them, we had to reject the data and repeat the measurement.

We identified two pattern beats immediately before the tested beat, the tested beat, and the beat immediately after the tested beat. We knew all coordinates of the important ventricle impulses from this point and we could find amplitudes of aBP and OS in both pattern beats and the tested beat (see Fig. 1).

Many external factors influenced the time course of OS. We have estimated two quality criteria which served for rejection of those examinations, during which the pulse oxygen saturation signal was not stable enough. We used them during both ways of the measurement. The first criterion assessed the stability of the magnitude of OS pulse amplitude. The measurement was accepted when the pulse amplitude of the first pattern beat was within $96 \%$ and $105 \%$ of the amplitude of the second pattern beat. The second criterion assessed stability of the level of the minimal saturation measured. The examination was accepted if the minimal level of the first pattern beat was within $-3 \%$ and $+4 \%$ of the amplitude of the second pattern beat above the minimal level of the second pattern beat.

\section{Measurement procedures}

\section{Dual chamber pacing with an AV interval change}

We changed the AV interval in steps of $50 \mathrm{~ms}$, from 50 $\mathrm{ms}$ to $100 \mathrm{~ms}, 100-150,150-200$, and from $200 \mathrm{~ms}$ to 250 $\mathrm{ms}$ and then from $250 \mathrm{~ms}$ to $200 \mathrm{~ms}, 200-150,150-100$, and from $100 \mathrm{~ms}$ to $50 \mathrm{~ms}$. We calculated an index of atrial contribution (6) (IAC) for each obtained data. The IAC is the difference between the amplitude of the tested beat and the amplitude of the second pattern beat expressed in \% of the amplitude of the second pattern beat. Accepted data together with the patient's name, the heart rate, and the time coordinates of the desired parts of the $\mathrm{aBP}$ and the OS curves were saved on hard disc into the text file. The file was automatically assigned its name with the number and the specification of the measurement procedure.

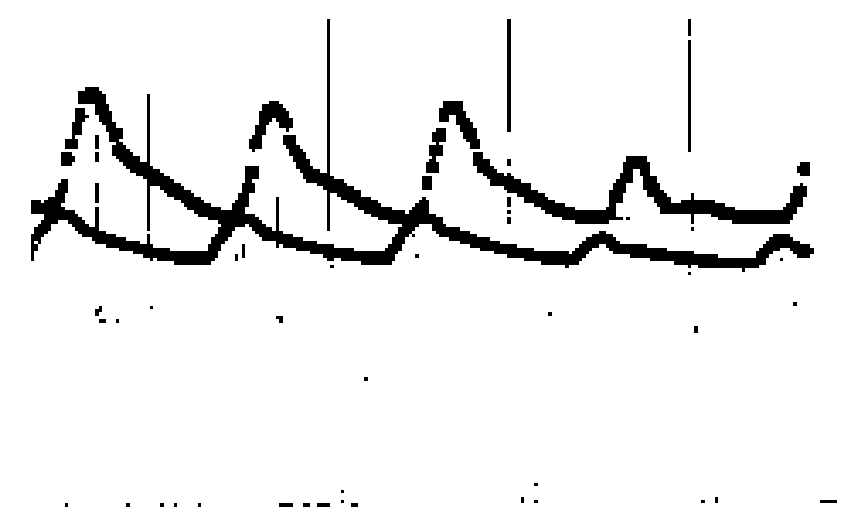

Fig. 1: Estimation of pulse amplitudes after the first and second pattern beats and the tested beat when switching the stimulation mode from DDIM to VVIM.

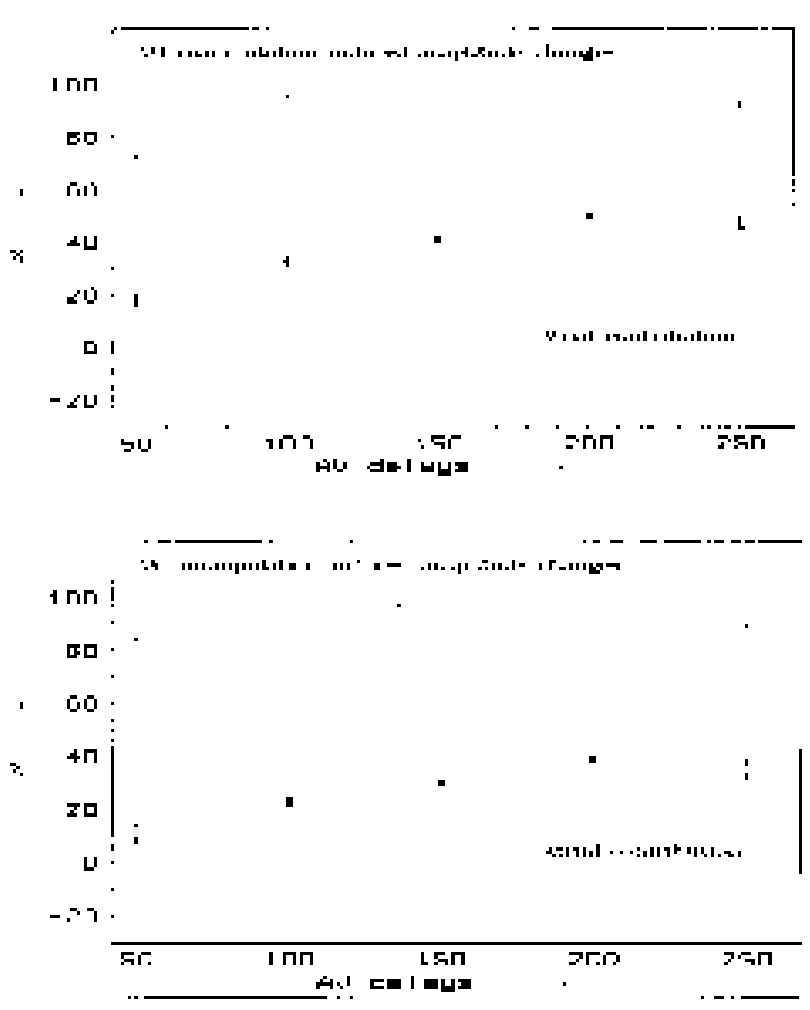

Fig. 2: Computer printout of one of the examinations. Values calculated from the oximetric signal (upper graph) and the aortic blood pressure signal (lower graph) using two different methods: The curves in the upper parts of graphs are constructed from the increments or decrements of the analyzed pulses, induced by changes in the AV interval length (the maximal amplitude was taken as $100 \%$ ). The points below are calculated from the changes induced by switching-off the atrial stimulus (switching from DDIM to VVIM of pacing). The maximal atrial contribution is found at the AV interval of $200 \mathrm{~ms}$. 


\section{Measurement with switching off the atrial impulse}

We switched the stimulation mode from DDIM to VVIM for 2-3 beats during the second procedure to estimate the optimal AVI. After switching off the atrial impulse the stimulation continued in DDIM. We performed the measurement at AVI's of 50, 100,150, 200, and $250 \mathrm{~ms}$. This sequence was measured two times. We attempted to locate a beat without an atrial impulse. Every variable was measured and data was saved the same way as in the first method of the measurement.

\section{Final analysis and the estimation of the optimal AV interval}

The data from the first procedure using DDIM pacing were multiply by -1 in measurement with the ascending changes of AVI. The final IAC for a given AVI was counted as the mean of indexes of atrial contribution from the ascending and the descending change of that AVI. The final IAC for a given AVI in the second measurement procedure was calculated as the mean of the indexes of atrial contribution measured for that specific AVI.

The graphic presentation was made after the determination of the optimal AV delay for aBP and OS as well. The beat with the maximal value of the final IAC was the beat in which the AC was maximal. The graphic presentation of the first procedure was displayed in the upper curve of the graph with the maximal AC being the $100 \%$ value. The decrements of $\mathrm{AC}$ for the other $\mathrm{AV}$ intervals were presented in $\%$ of the value of the preceding AC. The graphic presentation of the second measurement was placed in the lower part of graph. These points represented values of both indexes of $\mathrm{AC}$ and the final IAC in each AV interval (see Fig. 2).

\section{Results of the pilot study}

Twenty-four patients with Physios dual chamber pacemakers were enrolled into our pilot study. The pacemaker was implanted for sick sinus syndrome in 12 patients and for complete AV block in 12 patients. Both procedures of measurement were performed on each patient. Correlation between the values obtained for the aBP changes and oxygen saturation changes showed a tight association, the correlation coefficient being 0,982 for 432 measurements. From the aBP measurement, the optimal AVI was found to be $150 \mathrm{~ms}$ in 10 patients, $200 \mathrm{~ms}$ in 8 patients and $250 \mathrm{~ms}$ in 6 patients. The saturation pulse analysis identified the same interval in 19 patients. In the remaining 5 patients the maximal AC identified by the pulse saturation differed from the maximum identified by the pulse pressure analysis by no more than $50 \mathrm{~ms}$. In all of these 5 patients, the difference in magnitude of the IAC of the beats in question was less than $2 \%$ of the amplitude of the second pattern beat, i.e. the atrial contribution indexes of the two beats were nearly identical in amplitude. The mean AC that was determined by pulse oximetry in our study was $33,7 \%$ $(8,1-57,9 \%)$.

\section{Discussion}

Contemporary methods for optimizing the AV intervals are not routinely used. They are generally time consuming, invasive, and/or expensive. The most frequently used method for estimating an optimal AV interval is to evaluate the systolic volumes in different AV delays using echo Doppler measurement of the blood flow in the ascending aorta. One of disadvantages of this method is the high dependency on the experience level of echocardiographist (2). The other, more exact methods are invasive or use technical devices, which would not be common equipment in pacing laboratories $(3,4,5)$.

The pulse oximetry has not been routinely used for measurements of hemodynamic variables because it is only a relative value with big interindividual variability. The measurement of pulse oximetry is based on the photoplethysmographic principle using red and infrared light. The main aim of the development engineers was to get a digital form of the oxygen saturation value. The curve of the time course of the oxygen saturation changes is only a side product, complementing information about vital signs already available on the screen of the monitor. Our experience showed that the Marquette USA system is suitable for our new method. It shows the full amplitude and variations of the OS without loosing the minima by replacing them with an 0 value.

As documented in the literature, each of our patients had only one AV interval in which the atrial contribution was maximal (4). The time course of blood pressure values during the changes in stimulation was intraindividualy repeatedly constant.

The most important result was the evidence of the tight correlation between the changes in pressure pulse amplitude and changes in the pulse amplitude of oximetric signal during changes in the stimulation. The correlation coefficient 0,982 from our pilot study is similar to that from the Fargel and Lindvall study $(r=0,53-0,97)$. The value of atrial contribution estimated by our method is lower than the value estimated by Fargel (35-94\%).

The fact is important, since the same optimal AV interval in each patient was obtained by both procedures of measurement. This suggests that one could use only one procedure for estimating the AVI in the future. It seems, that the procedure in which the atrial impulse is switched off will be better, because the values obtained from the repeated measurements are similar.

Our new method has limitations as well. In addition to the construction of the pulse oximetry device, there is an important role of patient's compliance. The correct way to breath must be practiced with patients before measurements are recorded. The most frequent mistake of the patients was deeper inspiration before expected apnea or forced expirium immediately before apnea. Another mistake was Valsalva within apnea. Not all patients were able to avoid these mistakes. Movement of hands or fingers with the pul- 
se oxygen probe was another source of the measurement inaccuracies. These factors that affect the measurement were if the patient was nervous or there was an impact of a brain atherosclerosis and a parkinsonism.

\section{Conclusion}

If no other limitations will be found in the future, the present method would be a new, easy way for the optimization of AV interval in dual chamber paced patients.

\section{Acknowledgement}

Supported by grant No. 3677-3/96 of the Internal Grant Agency of the Ministry of Health of the Czech Republic.

\section{Literature}

1.Haskell RJ, French WJ. Physiological importance of different atrioventricular intervals to improved exercise performance in patients with dual chamber pacemakers, $\mathrm{Br}$ Heart J 1989;61:46-51.

2.Nishimura RA, Hayes DL, Holmes DR, Tajik AJ. Mechanism of hemodynamic improvement by dual-chamber pacing for severe left ventricular dysfuction: an acute Doppler and catheterization hemodynamic study. JACC 1995;25: 281-8.

3.Mitchell JH, Gupta DN, Payne RM. Influence of atrial systole on effective ventricular stroke volume, Circ Res 1965;17:11-8

4.Fargel B, Lindvall K. Non-invasive beat-to-beat analysis of stroke volume and digital pulse volume in patients with compete heart block and artificial pacing. Acta Med Scand 1979;205:185-90.
5.Kvasnička J, Tauchman M, Kovář D, Jandík J, Gajdošová I, Rejchrt P. Atrial contribution to ventricular ejection in sequentially paced patients. Physiol Res 1996;45:159-63.

6.Yellin EL, Sonnenblick EH, Frater RWM. Dynamic determinants of left ventricular filling: an overview. Cardiac Dynamics. Hague:Martinus Nijhoff Publishers, 1980:145-58. 7.Dvořák L, Sobotka M, Ledvinka P. Famulus 3.5 - příručka uživatele (user's manual), Jihlava:Wimpy, 1992.

8.Warner HR, Swan HJC, Connoly DC, Tompkins RG, Wood EH. Quantitation of beat-to-beat changes in stroke volume from the aortic pulse contour in man. J Appl Physiol 1953;5:495-505.

9.Warner HR, Rutishauser W. Comparison of beat-to-beat estimates of stroke volume from aortic pressure with flowmeter estimates (abstr.). Physiologist 1965;8:299.

10.Eliakim M, Sapoznikov D, Wienman J. Assessment of the atrial contribution to cardiac performance by a noninvasive photoplethysmographic technique, Cardiology 1973;58:7-13.

11.Kvasnička J, Měštan M, Tauchman M et al. Síňový příspěvek a optimální hodnota $\mathrm{AV}$ intervalu u sekvenčně stimulovaných nemocných, Vnitr Lek 1997;43:340-4.

Submitted May 1998.

Accepted July 1998.

MUDr. Miroslav Měštan, First Department of Internal Medicine, Charles University, Faculty of Medicine and Teaching Hospital, 50005 Hradec Králové, Czech Republic. 